\title{
La transparencia de la valoración documental en las administraciones autonómicas españolas
}

\author{
Ascensión de la Horra Márquez* \\ *Mancomunidad Comarca de la Sidra, Nava (Asturias). \\ Correo-e: ascenhorra@gmail.com | ORCID iD: https://orcid.org/0000-0002-8976-7747
}

Recibido: 24-09-19; 2a versión: 18-03-20; Aceptado: 23-03-20. Publicado: 18-02-21

Resumen: Este artículo analiza la situación de la valoración documental en la administración de las diferentes comunidades autónomas de España, a través del estudio de los documentos generados en el proceso de la valoración que se publican en sus páginas de archivos. Su objetivo es conocer el grado de desarrollo de los sistemas de valoración en las respectivas comunidades autónomas y si esos sistemas son transparentes y publican de forma activa sus resultados. En primer lugar, se presenta el marco conceptual de la valoración documental y se identifican los documentos que se generan en el proceso. Después, se analiza si esos documentos de la valoración están publicados en las páginas de archivos para presentar el grado de desarrollo de la valoración documental en las Comunidades Autónomas y su transparencia. Finalmente, se proponen unas recomendaciones generales para conseguir mejorar la información existente en la web sobre la valoración documental.

Palabras clave; administraciones públicas; archivística; archivos; Comunidades Autónomas; documentos; España; gestión de documentos; patrimonio documental; transparencia; valoración documental.

\section{Documentary appraisal transparency in the Spanish Autonomous Administrations}

Abstract: This paper analyzes the appraisal situation in the administration of the different Autonomous Communities from Spain, through the study of the generated documents in the appraisal process, that are published in its archives pages. Its objective is to know the development degree of the appraisal systems in the respective Autonomous Communities and if those systems are transparent and their results are actively published. First, the conceptual framework for documentary appraisal is presented and the documents generated in the process are identified. Then, it is analyzed whether these valuation documents are published in the archives pages, to present the development degree of the documentary appraisal in the Autonomous Communities and their transparency. Finally, some general recommendations are proposed to improve the existing information on the web about the documentary appraisal.

Keywords: appraisal; archival science; archives; Autonomous Communities; documentary heritage; documents; public administration; records management; Spain; transparency.

Cómo citar este artículo/Citation: De la Horra Márquez, A. (2021). La transparencia de la valoración documental en las administraciones autonómicas españolas. Revista Española de Documentación Científica, 44 ( 1), e 285. h ttps://doi.org/10.3989/ redc.2021.1.1716

Copyright: (c) 2021 CSIC. Este es un artículo de acceso abierto bajo los términos de la licencia de uso y distribución Creative Commons Reconocimiento 4.0 Internacional (CC BY 4.0). 


\section{INTRODUCCIÓN}

La valoración documental es una función archivística crucial ya que es el proceso por el que se decide qué documentos de las organizaciones se deben conservar y cuáles se deben eliminar. El resultado de sus decisiones determinará las fuentes que permitan a las generaciones futuras tener una visión fiel de la sociedad en la que han vivido. Sin embargo, la valoración no tiene como única finalidad expurgar o eliminar documentos, como tradicionalmente se ha entendido. Esta sería una visión parcial de la valoración, pues aunque, efectivamente, el resultado de la valoración pueda ser el expurgo o eliminación de documentos, la valoración es mucho más.

Su origen está íntimamente unido al desarrollo de la gestión de documentos o records management que se define como el "conjunto de tareas y procedimientos orientados a lograr una mayor eficacia y economía en la explotación de los documentos por parte de las organizaciones" (Cruz-Mundet, 2006). La facilidad de producir y reproducir documentos unida al desarrollo de los estados democráticos, caracterizados por la rendición de cuentas y la transparencia, sustentados en gran medida por los documentos, han producido una inflación documental que ha hecho necesario un cambio de modelo.

Se ha pasado de considerar la eliminación de documentos como antiarchivística (Lodolini, 1984), a entender que la producción actual de documentos ha crecido en tal proporción que es imprescindible su gestión. Hoy está aceptado por la comunidad archivística que no se puede conservar todo lo que se produce y hay que prescindir de lo inútil para concentrar los recursos económicos, personales y logísticos en conservar los documentos que nos ayuden a construir la memoria colectiva.

Debido a la transcendencia de esta función archivística, fundamentalmente por lo que supone de destrucción de patrimonio documental, se han establecido en las diferentes organizaciones públicas sus respectivos sistemas de valoración. En España el camino se inicia con la entrada en vigor de la Ley de Patrimonio Histórico Español (España, 1985) que establecerá los principios básicos sobre los que se inspirarán el resto de administraciones.

Este artículo analiza la situación actual de la valoración documental en la administración de las diferentes Comunidades Autónomas (CC. AA.) de España, a través del estudio de los documentos generados en el proceso de la valoración que se publican en las web de archivos. Entre los objetivos principales está conocer el grado de desarrollo de los sistemas de valoración en las respectivas comunidades autónomas y conocer si esos sistemas son transparentes y publican de forma activa sus resultados.

La oportunidad del artículo se encuentra en la escasez de estudios descriptivos-comparativos sobre los procesos de valoración documental llevados a cabo por las diferentes administraciones públicas españolas, y en concreto por las CC.AA. Se reconocen limitaciones en el artículo pues la única fuente utilizada para la toma de datos ha sido la web y, aunque se da por hecho que todo está en la web, no siempre es así. Otros problemas reseñables son que la información disponible podía no estar actualizada y que, a pesar de haber realizado búsquedas exhaustivas, no se haya localizado toda la información útil para la investigación.

\section{MARCO TEÓRICO}

Valorar significa juzgar el valor de algo, en nuestro caso el valor de los documentos. Los criterios modernos que rigen la valoración documental fueron sistematizados y difundidos por la archivística norteamericana y en concreto por la obra de Schellenberg (1958), que estableció unos principios que permanecen en esencia en todos los sistemas de valoración del mundo. Es lo que se conoce como la teoría de los dos valores de los documentos: valor primario y valor secundario.

El valor primario es aquel que va unido a la finalidad inmediata por la cual el documento se ha producido por una organización e identifica tres componentes: el valor administrativo, el jurídico y el fiscal. El valor secundario es aquel que obedece a otras motivaciones que no son la propia finalidad del documento, tales como el valor histórico o testimonial de la organización y el valor informativo.

En la última década del siglo $X X$ se produjeron nuevas aportaciones a la teoría de la valoración documental conocidas como macrovaloración o valoración funcional. Uno de sus exponentes es Terry Cook, de los Archivos Nacionales de Canadá, que establece un nexo entre la importancia del organismo creador y el valor de los documentos producidos (Couture, 2003). Aporta un enfoque estratégico en la planificación de la valoración estableciendo dos fases. La primera, la macrovaloración, en la que se estudian las razones por las que el documento existe, los cometidos y funciones del creador y se establecen prioridades en las unidades administrativas a estudiar de "arriba abajo" dentro de la organización. Y una segunda fase, la microvaloración, en la que se juzga el valor primario y secundario de los documentos. 
En España el interés por la valoración se inicia en los años 80 cuando Cortés Alonso (1983), siguiendo a Schellenberg, trasciende la teoría de los valores a las series documentales aplicando a éstas la valoración. Desde entonces, la teoría de la valoración se estudiará en los diferentes manuales de archivística como los de Heredia Herrera (1986, 2013) y Cruz Mundet (1994, 2006, 2012). A estos se unirán las monografías que profundizan en el estudio teórico y metodológico de la valoración documental, como las obras de Vázquez Murillo (1995), La Torre Merino (2000), Hernández Olivera y Moro Cabero (2002b) o Cermeno Martorell y Rivas Palá (2010).

La publicación en 2016 de la segunda edición de la norma UNE-ISO 15489 (UNE-ISO, 2016) dará un impulso teórico de la valoración a nivel internacional y nacional, mejorando la primera edición de la norma del año 2001, publicada en España en 2006 (UNE-ISO, 2006). En ella la valoración documental, junto con la identificación, aparecerá como el proceso nuclear para el funcionamiento de los sistemas de gestión de documentos y sus objetivos se amplían al "diseño e implementación de sistemas de gestión documental, el desarrollo de políticas y procedimientos, la definición de requisitos de metadatos y el desarrollo de instrumentos de gestión de documentos, como calendarios de conservación o reglas para acceso y permisos" (UNE-ISO, 2016). Además establece que el proceso de identificación y valoración se deberá documentar para recomponer y entender las acciones que se realizaron, el porqué, el cómo y el cuándo. Y por supuesto, por transparencia y aplicación del buen gobierno.

Esta visión de la valoración se confirma con la ISO/TR 21946:2018 Information y Documentation: Appraisal for managing records (ISO/TR, 2018), que desarrolla el proceso de valoración en el marco de la gestión de documentos establecido por la UNE ISO 15489. En esta nueva norma ISO se proporciona orientación sobre cómo llevar a cabo el concepto de valoración descrito en la UNE ISO 15489-1 y dibuja el proceso técnico como un ciclo continuo compuesto por cuatro grandes actividades: recopilación de información y análisis; valoración e implementación; vigilancia; revisión y acción correctiva (Fernández-Cuesta, 2018).

Paralelamente, se publicarán también artículos sobre la teoría y metodología de la valoración (Hernández-Olivera, 2005 y Hernández-Olivera y Moro-Cabero, 1997 y 2009), o sobre la valoración como herramienta para crear la memoria social (Hernández-Olivera, 2013). También sobre la valoración del documento electrónico (Hernández-Olivera, 2008 y Rivas-Palá, 2013), la relación de la valoración y la transparencia (Casellas i Serra, 2017a) o propuestas basadas en el análisis funcional de las organizaciones y que aportan criterios y elementos para la valoración (Casellas i Serra, 2014, 2017b).

La visión práctica de la valoración, con ejemplos de su aplicación, la encontramos por primera vez en las ponencias de las VII Jornadas de Archivos Municipales de Madrid (Grupo de archiveros de Madrid, 1990) con el título El expurgo en los archivos municipales, propuesta de un manual, que presentan una propuesta de cuadro de clasificación municipal con la valoración de cada una de sus series. Otros encuentros profesionales han ahondado en la teoría de la valoración pero, sobre todo, en la exposición de las experiencias en toda clase de archivos y sobre toda clase de tipos de documentos. Ahí están las I Jornadas sobre Metodología para la Identificación y Valoración de Fondos Documentales de las Administraciones Públicas (Ministerio de Cultura, 1991) o las 6a Jornadas Archivando de la Fundación Sierra Pambley (González-Cachafeiro, 2013).

Existen publicaciones que muestran cómo se ha realizado la valoración de documentos en diferentes comunidades autónomas como la de Castilla y León (Garmendia-Ruiz, 2000; Hernández-Olivera y Moro-Cabero, 2003), Cataluña (Cermeno-MartoreII, 2003) y Andalucía (Páez-García, 2010), o en la Administración General del Estado (Hernández-Olivera y Moro-Cabero, 2002a). También sobre cómo se valora un determinado tipo de series documentales (Casellas i Serra, 2001; Rivas-Palá, 2003) o sobre la valoración en diferentes tipos de archivos, como los judiciales (Fernández-Cuervo, 1996; Gómez-Loeches, 1997).

Sin embargo, se han encontrado pocos trabajos de investigación que estudien la valoración con una visión de conjunto de todas las Comunidades Autónomas españolas. Uno de ellos es el de las XIX Jornadas de Archivos Municipales de Madrid (Grupo de Archiveros Municipales de Madrid, 2013), que presenta un estudio legislativo comparado sobre la metodología de valoración y selección documental de las diez y siete Comunidades Autónomas con enlaces a los calendarios de conservación o tablas de valoración en un trabajo recopilatorio. Entre sus conclusiones destaca que la implantación de los sistemas de valoración documental es muy desigual entre las distintas Comunidades Autónomas.

Otro es el artículo de Cascudo Rasilla (2018) que repasa los diferentes modelos de sistemas de valoración autonómicos y presenta un estudio cuantitativo de sus resultados, mediante el recuento de tablas o calendarios de valoración o conservación 
publicados en los respectivos boletines oficiales autonómicos.

Para establecer el marco teórico y la justificación del artículo, se ha realizado un análisis de las fuentes documentales secundarias. Se han utilizado para la búsqueda de recursos o fuentes de información las principales bases de datos bibliográficas (Dialnet, ISOC, WoS, Scopus, LISTA, AENOR), repositorios (E-Lis y Recolecta), y portales institucionales (BOE, Portal de Administración Electrónica, Portal de Archivos del Ministerio de Cultura, Portales de Archivos de las CC.AA. y páginas web de Comisiones Calificadoras de Documentos de las CC.AA). La palabra clave de las búsquedas fue el término aceptado en el Tesauro de Biblioteconomía y Documentación editado por el CINDOC (Sorli-Rojo y Mochón-Bezares, 2002), "valoración documental", pero, debido a la escasa normalización de la terminología archivística, también se han utilizado para las búsquedas otros términos sinónimos "selección documental", "eliminación documental", "evaluación documental" o "expurgo". Se han seleccionado monografías, normativa española y recursos web sin límite de fecha y los artículos de revistas, preferentemente de menos de 10 años de antigüedad y todos limitados a España.

\subsection{La valoración documental}

Según el Diccionario de Terminología Archivística la valoración documental es la "fase del tratamiento archivístico que consiste en analizar y determinar los valores primarios y secundarios de las series documentales, fijando los plazos de transferencia, acceso, y conservación o eliminación total o parcial" (Ministerio de Cultura, 1995). Años más tarde, la UNE-ISO 15489:2016, 7.1, publica una definición de valoración, junto a la identificación, en la que se ofrece una visión desde la perspectiva de la gestión de documentos y "será el proceso que evalúe las actividades de una organización para determinar qué documentos necesitan crearse y capturarse, y por cuánto tiempo es necesario conservarlos" (p. 17). Es decir, sitúa la valoración como un proceso archivístico que debe iniciarse antes de la creación de los documentos, por lo que debe realizarse en la fase de diseño y producción de documentos e introduce una visión sistémica de la gestión de documentos que cambia la visión finalista de los archivos.

Esta nueva visión fundamenta el desarrollo de la administración electrónica y la obligación de plasmar los actos administrativos en documentos y expedientes electrónicos (España, 2007a y 2015). Por primera vez en la legislación española se establecerán como obligatorios procesos archivísticos que hasta entonces formaban parte del corpus teórico de la archivística pero no estaban integrados en ninguna normativa legal de obligado cumplimiento. La valoración será un proceso fundamental que, en la nueva normativa, se incluirá dentro de un proceso más amplio denominado calificación, que es un término que ya aparece en la Ley de Patrimonio Histórico Español (España, 1985, art. 58), y que se define en la guía de aplicación de la Norma Técnica de Interoperabilidad (NTI) de Política de gestión de documentos electrónicos (MINHAP, 2016b) como:

Proceso de gestión de documentos que tiene por finalidad, en base a un análisis de los valores de los documentos, establecer los plazos de permanencia de los documentos en el sistema de gestión, de transferencia y eliminación en su caso, así como los plazos de acceso y la eventual calificación como documento esencial de una organización (p. 38).

En este artículo no se analizará el régimen de acceso de los documentos.

\subsection{Sistemas de valoración y su documenta- ción}

Se debe entender la valoración documental como una función que se lleva a cabo dentro de un sistema archivístico, formado por unas normas reguladoras y unos órganos, centros y servicios competentes en la gestión de los documentos y de los archivos (España, 2011). Todas las CC.AA. han ido creando sus sistemas archivísticos mediante su normativa correspondiente.

Pero para poder llevar a cabo los procesos de valoración en las organizaciones también es imprescindible, como señalan Cermeno y Rivas (2011), que se creen sistemas de valoración dentro de esos sistemas archivísticos. Estos deberán tener los siguientes elementos:

- La normativa de creación del sistema y del procedimiento para la aprobación de acuerdos de valoración a partir de unos criterios que se establezcan.

- Los órganos que dictaminen sobre la valoración. Son las llamadas comisiones calificadoras de documentos que "son el instrumento reglamentario del que se dota la administración para la función de evaluar y fijar los plazos de conservación de los documentos administrativos públicos" (Ruiz-Cagigal, 2017). Éstas generan documentos con los nombramientos de sus miembros y su composición, las convocatorias y órdenes del día y las actas de sus reuniones.

- Los órganos que aprueben los acuerdos de valoración, mediante resolución administrativa y 
publicación en el boletín oficial correspondiente. Generalmente este órgano es la consejería con competencias en archivos.

- Los recursos personales, en su gran mayoría archiveros, que inician, impulsan, documentan y ejecutan los acuerdos.

- Los documentos generados en el proceso de valoración que deberían ser al menos los siguientes, además de los mencionados en los puntos anteriores: los estudios y registros de series identificadas y valoradas, los calendarios de conservación con los dictámenes sobre conservación o eliminación y acceso, los listados de documentos esenciales, los calendarios de transferencia de las series documentales a conservar y enviar a los archivos, y, muy importantes, las actas y registros de eliminación de la documentación que no se conserva.

\subsection{La transparencia de la valoración docu- mental}

En este estudio se quiere analizar no sólo los procesos de la valoración documental, sino también su transparencia comprobando qué información de la valoración se publica en las páginas de archivos.

Las leyes de transparencia, estatales y autonómicas, han reforzado la obligación legal de hacer pública y accesible la información institucional, organizativa y de planificación, de relevancia jurídica y de naturaleza económica, presupuestaria y estadística de las administraciones públicas (España, 2013). En este contexto, la destrucción de documentos administrativos, como una de las consecuencias de la valoración, puede interpretarse como contraria al deber de conservación del patrimonio documental y al derecho de acceso de los ciudadanos a esos documentos. Por tal motivo, como señala Casellas i Serra (2017a), es tan importante la transparencia en el proceso, para ofrecer las máximas garantías de seguridad jurídica con una sólida base legal y un procedimiento lo más objetivo posible.

Pero, ¿dónde se publica la información de la valoración? Pueden existir varias opciones, la primera de ellas son las páginas o portales de archivos. En ellas, la información de valoración puede estar reunida dentro de una sección propia, o bien puede haber información mezclada con otros contenidos de los sistemas de archivos.

Otra opción son los portales de transparencia. La valoración documental es un proceso que afecta a la organización interna de las administraciones públicas y a su patrimonio documental. El Índice de Transparencia INCAU (Transparency International
España, 2016) de los portales de transparencia de las CC.AA. evalúa positivamente que se haga pública la "política o la forma de llevar a cabo la gestión, la conservación y la eliminación de los documentos y archivos", por eso se publica información de valoración en estas páginas.

La información estadística es también otra opción para la transparencia del trabajo de los sistemas de archivos y pueden aportar datos cuantitativos sobre valoración documental, aunque, como señala Pérez Santana (2018), hace falta una normalización de metodologías, indicadores, etc.

Aparte de todas estas opciones está la obligación de publicación de los calendarios de conservación en los boletines oficiales de las CC.AA. Éstos son los documentos que recogen los dictámenes sobre conservación o eliminación de los documentos de un organismo. Esta circunstancia deviene de la obligación de publicidad de los actos administrativos, como son las resoluciones por las que se aprueban, modifican o eliminan los calendarios de conservación dictados por los órganos gubernativos correspondientes, en este caso, las consejerías competentes en materia de archivos.

La forma en que se publica la información sobre la valoración también influye en la transparencia. La accesibilidad y la reutilización son dos principios técnicos a seguir como establece la Ley de transparencia, acceso a la información pública y buen gobierno en su artículo 11 (España, 2013). También, que la información esté actualizada y que se nos informe de la fecha de su publicación. La accesibilidad tiene que ver con que la información se proporcione estructurada para facilitar la identificación y búsqueda de la información. La reutilización con que la información se publique en formatos que permitan su uso por personas físicas o jurídicas, con fines comerciales o no comerciales, de acuerdo con lo previsto en la legislación vigente (España, 2007b).

También es importante que la información publicada tenga sistemas de búsquedas o sistemas de ordenación para facilitar su recuperación, elementos que deben aplicarse, entre otros, en los sistemas de consulta (Codina-Bonilla, 2018).

\section{OBJETIVOS}

El objetivo de este artículo es analizar cómo se está llevando a cabo el proceso de la valoración de documentos en la administración de las comunidades autónomas españolas y cómo se está difundiendo todo su trabajo, tomando como base el análisis de la documentación generada en su desarrollo y publicada en sus respectivas páginas web de archivos. 
Este objetivo principal se concreta en los siguientes objetivos específicos:

- Analizar la documentación generada por la valoración documental que se publica en las páginas web de archivos de las CC.AA.

- Analizar dónde y cómo se publica esa documentación en las páginas web institucionales.

- Estos objetivos se concretan en las siguientes preguntas de investigación:

- ¿Se publica la normativa de la valoración documental en las páginas web de archivos de las CC.AA.?

- ¿Y la documentación producida por las comisiones calificadoras?

- ¿Y la documentación del proceso de valoración?

- ¿Y la de aplicación de la valoración?

- ¿En qué páginas web, además de las de archivos, se publica la documentación de la valoración?

- ¿Cómo se publican los documentos que contienen la información sobre la valoración en las páginas web?

\section{METODOLOGÍA}

Para la consecución de los objetivos expuestos se ha propuesto la realización de un estudio descriptivo-comparativo, documental, transversal y mixto.

Se utiliza una metodología descriptiva-comparativa por el alcance de los conocimientos que aporta, documental por los medios utilizados para obtener los datos, transversal pues refleja la situación de un momento concreto que coincide con el periodo de recogida de datos y mixta pues se recolectan, analizan y combinan datos cuantitativos y cualitativos.

\subsection{Definición del universo}

La unidad de análisis de la investigación han sido las páginas web de archivos de las diez y siete Comunidades Autónomas españolas y los portales de transparencia de las mismas. No se ha seleccionado ninguna muestra, pues el objetivo del estudio es analizar todas y cada una de las páginas web para ofrecer una visión del conjunto del territorio español.

En este artículo se entiende como sede o página web lo que Codina Bonilla (2000) define como "una unidad virtual, en tanto que digital, más o menos arbitraria, que puede estar formada por diversos documentos, subsecciones, cada uno de los cuales puede tener su propia URL". Es decir, un conjunto de páginas web relacionadas y propias de un domi- nio o subdominio de la misma institución, en nuestro caso, de una CC.AA. Para las denominaciones de las CC.AA. se ha tomado como referencia el INE (Instituto Nacional de Estadística).

\subsection{Recogida y tratamiento de datos}

La técnica de investigación utilizada para la recogida de datos ha sido la observación y análisis de los contenidos de las diferentes páginas web de las diez y siete CC.AA., con el fin de localizar la información necesaria para los objetivos del estudio. La búsqueda de datos se realizó entre febrero y junio de 2019.

El primer paso fue buscar las páginas de los sistemas de archivos de las consejerías competentes de las CC.AA. y analizar su contenido en busca de información sobre valoración documental. Se identificó dónde estaba publicada la información dentro de la estructura orgánica de las CC.AA., se buscaron los documentos del proceso de valoración documental y, por último, se analizó cómo aparecían esos documentos en las webs, atendiendo a su accesibilidad, reutilización, actualización y sistemas de consulta empleados. A continuación, se analizaron las páginas web de transparencia de las CC.AA. para comprobar si ofrecían o no información sobre la valoración documental, y si la ofrecían, analizar qué tipo de información.

Para la recogida de los datos cuantitativos se ha utilizado una tabla diseñada ad hoc en el programa Excel de Microsoft, para su posterior tratamiento estadístico. Para los datos cualitativos se han elaborado tablas en Word de Microsoft.

Para establecer el listado de indicadores se han tomado como referencia los trabajos de Cermeno y Rivas (2010 y 2011) y de Hernández y Moro (2002b). Ambos ofrecen una exhaustiva información sobre los documentos que deben generarse en el proceso de la valoración.

Los indicadores a analizar se agrupan en seis epígrafes:

1. Documentación normativa: sobre la creación del sistema de valoración, el funcionamiento de las comisiones calificadoras y los criterios de valoración.

2. Documentación de las comisiones calificadoras: nombramientos y composición, convocatorias y órdenes del día, actas de las reuniones de la comisión.

3. Documentación del proceso de valoración: listado/registro de series valoradas, estudios de identificación y valoración, calendarios de conservación, formularios, listados de documentos esenciales. 
4. Documentación de la aplicación de la valoración: actas de eliminación, registros de eliminación, calendarios de transferencias.

5. Identificación de las webs donde se localizan los documentos de la valoración: páginas de los sistemas de archivos de las CC.AA., páginas propias de las comisiones calificadoras, portales de transparencia.

6. Cómo se presenta la información: formatos reutilizables, información estructurada o tablas, información a través de enlaces, sistemas de búsqueda, sistemas de ordenación, fecha de actualización de los datos.

\section{RESULTADOS}

Después de extraer la información de las diez y siete CC.AA. a través del análisis de sus sedes o páginas web de archivos se analizaron los datos para dar respuesta a los objetivos planteados.

En la Tabla I se señalan, mediante la $X$, los documentos de valoración documental publicados en las webs de archivos de cada una de las CC.AA., ordenadas de las que más documentos publican a las que menos.

\subsection{Documentación normativa}

El resultado del análisis muestra que diez de las diez y siete CC.AA., es decir, el $59 \%$ del total, publican en sus web de archivos la normativa de creación del sistema de valoración. Esta normativa de creación del sistema debe completarse con la normativa que regule el funcionamiento de los órganos consultivos y el procedimiento que se seguirá para la valoración documental. El resultado del análisis muestra

Tabla I. Documentos de valoración documental publicados en las webs de archivos de las Comunidades Autónomas.

\begin{tabular}{|c|c|c|c|c|c|c|c|c|c|c|c|c|c|c|}
\hline & \multicolumn{3}{|c|}{$\begin{array}{l}\text { DOCUMENTOS } \\
\text { NORMATIVA }\end{array}$} & \multicolumn{3}{|c|}{$\begin{array}{l}\text { DOCUMENTOS } \\
\text { COMISIONES }\end{array}$} & \multicolumn{5}{|c|}{$\begin{array}{l}\text { DOCUMENTOS PROCESO } \\
\text { VALORACIÓN }\end{array}$} & \multicolumn{3}{|c|}{$\begin{array}{l}\text { DOCUMENTOS } \\
\text { APLICACIÓN } \\
\text { VALORACIÓN }\end{array}$} \\
\hline & 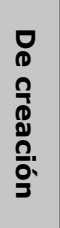 & 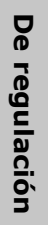 & 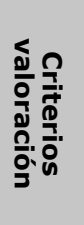 & $\begin{array}{l}2 \\
\frac{1}{3} \\
\frac{3}{0} \\
\frac{6}{0} \\
\frac{n}{0} \\
\frac{3}{3}\end{array}$ & 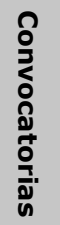 & 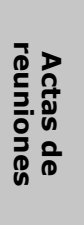 & 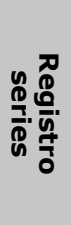 & 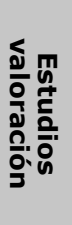 & 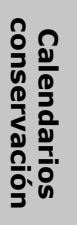 & 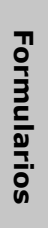 & 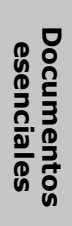 & 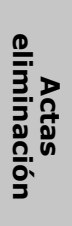 & 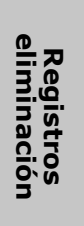 & 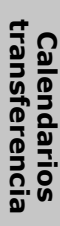 \\
\hline Cataluña & $X$ & $x$ & $X$ & $x$ & $X$ & $X$ & $X$ & - & $X$ & $X$ & - & - & $X$ & - \\
\hline Madrid, C. & $x$ & $x$ & $x$ & $x$ & - & $x$ & $x$ & $x$ & $x$ & $x$ & - & - & $x$ & - \\
\hline País Vasco & $x$ & $x$ & $x$ & $x$ & - & $x$ & - & - & $x$ & $x$ & - & - & - & - \\
\hline Andalucía & $x$ & $x$ & - & $x$ & - & $x$ & $x$ & - & $x$ & $x$ & - & - & - & - \\
\hline C. Valenciana & $X$ & $x$ & - & $x$ & - & - & $x$ & $x$ & $x$ & $x$ & - & - & - & - \\
\hline Canarias & $X$ & $x$ & $X$ & $x$ & - & - & $x$ & - & $x$ & - & - & - & - & - \\
\hline Extremadura & $x$ & $x$ & $x$ & - & - & - & $x$ & $x$ & $x$ & - & - & - & - & - \\
\hline Castilla La Mancha & - & $x$ & - & $x$ & - & $x$ & - & - & $x$ & $x$ & - & - & - & - \\
\hline Aragón & $X$ & - & - & $x$ & - & - & $x$ & $x$ & $x$ & - & - & - & - & - \\
\hline Balears, I. & - & $x$ & - & - & - & - & $x$ & $x$ & $x$ & $x$ & - & - & - & - \\
\hline Galicia & - & - & $x$ & - & - & - & $x$ & $x$ & $x$ & $x$ & - & - & - & - \\
\hline Navarra, C.F. & $x$ & $x$ & - & - & - & - & $x$ & - & $x$ & $x$ & - & - & - & - \\
\hline Castilla y León & $X$ & $x$ & - & - & - & - & $x$ & - & $x$ & - & - & - & - & - \\
\hline Rioja, La. & - & $x$ & - & - & - & - & - & - & $x$ & - & - & - & - & - \\
\hline Murcia, R. & - & - & - & - & - & - & $x$ & - & - & - & - & - & - & - \\
\hline Asturias, $\mathrm{P}$. & - & - & - & - & - & - & - & - & - & - & - & - & - & - \\
\hline Cantabria & - & - & - & - & - & - & - & - & - & - & - & - & - & - \\
\hline
\end{tabular}


que doce de las páginas de archivos, es decir, el $71 \%$ del total, publica esta información en la web. Por último, dentro de la documentación normativa deben publicarse también los criterios básicos de conservación y eliminación que se tienen en cuenta para la valoración. Solo seis de las CC.AA., es decir, el $35 \%$ del total, publica en la web de archivos la información sobre los criterios de valoración. La información sobre la documentación normativa de cada una de las CC.AA. se puede observar en la Tabla I.

Se comprueba que los órganos consultivos que se crean mediante la normativa de las distintas CC.AA. reciben cada uno una denominación distinta produciéndose una gran falta de normalización terminológica, no obstante, en este artículo se utilizará el término comisiones calificadoras para referirnos a todos ellos.

\subsection{Documentación de las comisiones califi- cadoras}

El resultado del análisis muestra que ocho de las diez y siete CC.AA., es decir, el $47 \%$ del total, publican en sus webs de archivos información sobre los nombramientos de las comisiones y su composición. La Comunidad de Cataluña es la única que publica las convocatorias y órdenes del día de las reuniones de la comisión calificadora en su web. Las actas de las reuniones de las comisiones con la información sobre las interpretaciones o discusiones que motivan un dictamen u otro, solo se publican en cinco CC.AA., es decir, en el $29 \%$ del total. En la Tabla I se pueden identificar los datos sobre la documentación de las comisiones calificadoras de cada CC.AA.

\subsection{Documentación del proceso de valoración}

El resultado del análisis muestra que la primera información de valoración que se encuentra en las páginas de archivos es un listado, tabla o registro con los nombres de las series documentales valoradas. Estos se encuentran en catorce webs de las CC.AA., el $82 \%$ del total. La información sobre los documentos del proceso de valoración, también se pueden observar en la Tabla I.

Los estudios de identificación y valoración se muestran en seis de las diez y siete CC.AA., es decir, el $35 \%$ del total, y son documentos en Pdf o Word a los que se accede mediante enlaces desde las series del listado anterior. Se observa que estos estudios no contienen los mismos campos y tampoco utilizan los mismos términos para denominarlos. Además, unos son más exhaustivos, como, por ejemplo, los de la Comunidad de Madrid, y otros publican resúmenes, como en la Comunitat Valenciana.
Los calendarios de conservación son los documentos más publicados en las webs de archivos, catorce de las diez y siete CC.AA., el $82 \%$ del total. Los campos de información que contienen son muy similares entre ellos y pueden presentarse en la propia web, mediante enlaces a documentos Pdf o Word, o bien, mediante enlaces a los boletines oficiales de las comunidades donde es obligatoria su publicación. También se publican formularios para cumplimentar los estudios de identificación y valoración. En nueve webs, el 53\% del total, se publican formularios tipo, a veces con instrucciones para cumplimentarlos, para la presentación de estudios propuestas de identificación y valoración dentro del sistema de valoración documental establecido. Por último, se observa que ninguna CC.AA. ha publicado en sus webs de archivos información sobre los documentos esenciales.

\subsection{Documentación de la aplicación de la va- loración}

El resultado del análisis muestra que ninguna CC.AA. expone en sus webs de archivos las actas de eliminación de documentos, por lo que no se publica la información sobre el qué, el cómo, el dónde y el cuándo se destruye patrimonio documental de una organización. Los calendarios de transferencias tampoco se publican en ninguna página de archivos, si bien es cierto que son documentos que pueden entenderse como de gestión interna, pueden aportar información sobre dónde se localizan las fracciones de series dentro de un sistema de archivos. Solo la Comunidad de Madrid muestra en su página web un registro de eliminaciones que proporciona información sobre número de unidades de instalación de una serie documental autorizadas para su eliminación, los metros lineales que ocupan y la fecha de la aprobación de la eliminación. En Cataluña también publican los metros lineales de series eliminadas por organismo, aunque dentro de la Memoria anual de su Comisión Nacional de Acceso, Evaluación y Selección Documental (CNAATD), que está publicada en la página de archivos. Otras comunidades como Castilla La Mancha sí proporcionan información de eliminación de documentos, pero de forma cuantitativa a través de sus estadísticas.

\subsection{Comparativa de los documentos de valo- ración publicados en las páginas de archivos}

Para poder ofrecer una visión general del desarrollo de los procesos de valoración de las diferentes CC.AA. y de su nivel de transparencia, se hace un análisis comparativo de los resultados de los cuatro puntos anteriores, para comprobar cuántos 
Figura 1. Total de documentos de valoración publicados por Comunidades Autónomas

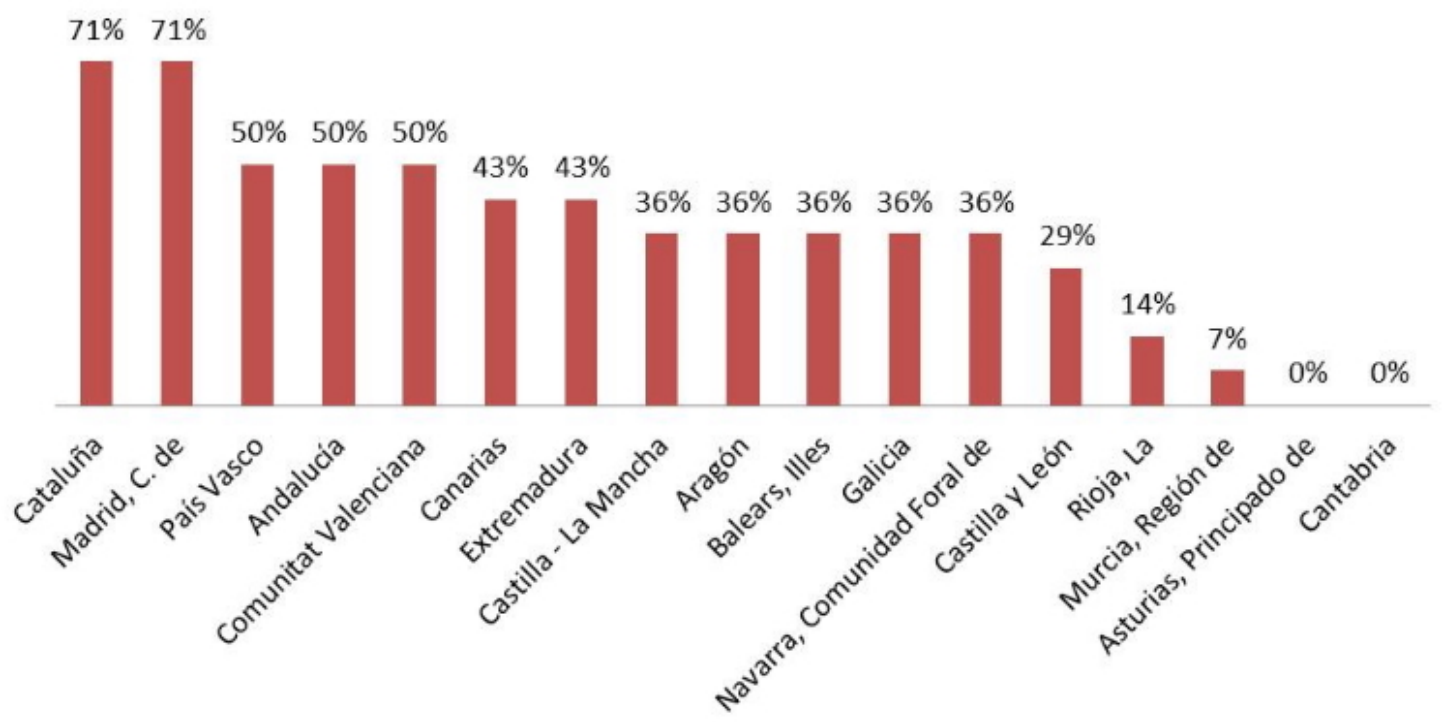

y cuáles documentos del proceso publican cada una en sus páginas de archivos.

En la figura 1 se muestra el porcentaje total de documentos del proceso de valoración que se publican en las páginas de archivos de las CC.AA.

Cataluña y la Comunidad de Madrid son las comunidades que más documentos publican en sus páginas de archivos, diez de los diez y seis documentos estudiados, el $71 \%$. Cataluña no publica los estudios de identificación y valoración, las actas de eliminación, los calendarios de transferencias y los documentos esenciales. Y la Comunidad de Madrid no publica las convocatorias a las reuniones de la comisión calificadora, y como Cataluña, tampoco publica las actas de eliminación, los calendarios de transferencias y los documentos esenciales. Les siguen el País Vasco, Andalucía y la Comunitat Valenciana con siete documentos respectivamente, el $50 \%$. Después le siguen el resto de comunidades en descenso hasta las que no publican ningún documento de valoración en sus webs de archivos.

\subsection{Páginas donde se publica la documenta- ción de valoración}

Tras un análisis exhaustivo de los portales institucionales de las CC.AA. para identificar en qué webs aparece la información sobre la valoración documental, se comprobó que la gran mayoría, en quince de las diez y siete comunidades, publicaban esta información en webs dependientes de las consejerías de cultura, lo que supone el $88 \%$ del total.
Y el resto, el 12\%, o la publican en la Consejería de Presidencia, como es el caso de Canarias, o en la Consejería de Administraciones Públicas y Hacienda, como sucede en La Rioja. En la siguiente Tabla II se identifican las páginas web donde las CC.AA. publican la documentación de valoración.

La mayoría de las CC.AA., siete de ellas, lo que supone el $41 \%$ del total, publican la información en las webs de las comisiones calificadoras de documentos creadas en cada sistema y recogen de forma exclusiva toda la información sobre valoración documental. Estas webs de las comisiones calificadoras se han considerado en los apartados anteriores del estudio, como webs de archivos, pero en éste apartado se ha decidido identificarlas para plasmar esta diferenciación que aparece en la estructura de las páginas de archivos de las CC.AA. Luego están los casos de otras Comunidades que, o bien publican la información en sus webs de archivos, cinco de las diez y siete, el $29 \%$, o publican la información repartida indistintamente entre las webs de las comisiones calificadoras y las webs de archivos, tres de ellas, el $18 \%$ del total. Por último, están aquellas que no publican información sobre valoración documental en sus webs de archivos.

También se han analizado las webs de transparencia de las CC.AA. De éstas, siete de las diez y siete CC.AA., es decir, el $41 \%$ del total, ofrecen información sobre valoración documental. Se observa que la información sobre valoración documental en los portales de transparencia es muy dispar. Algunas CC.AA. utilizan las estadísticas de 
Tabla II. Páginas Web donde se publica la documentación de valoración

\begin{tabular}{|l|c|c|c|c|}
\hline & $\begin{array}{c}\text { WEB COMISIÓN } \\
\text { CALIFICADORA }\end{array}$ & WEB ARCHIVOS & $\begin{array}{c}\text { REPARTIDO ENTRE } \\
\text { AMBAS WEBS }\end{array}$ & $\begin{array}{c}\text { WEB } \\
\text { TRANSPARENCIA }\end{array}$ \\
\hline Andalucía & $\mathrm{X}$ & - & - & $\mathrm{X}$ \\
\hline Aragón & - & - & - & - \\
\hline Asturias, P. & - & - & - & - \\
\hline Balears, I. & - & $\mathrm{X}$ & - & - \\
\hline Canarias & $\mathrm{X}$ & - & - & - \\
\hline Cantabria & - & - & $\mathrm{X}$ & $\mathrm{X}$ \\
\hline Castilla y León & - & $\mathrm{X}$ & - & - \\
\hline Castilla La Mancha & - & - & - & $\mathrm{X}$ \\
\hline Cataluña & $\mathrm{X}$ & - & - & $\mathrm{X}$ \\
\hline C. Valenciana & $\mathrm{X}$ & - & - & - \\
\hline Extremadura & $\mathrm{X}$ & $\mathrm{X}$ & - & $\mathrm{X}$ \\
\hline Galicia & - & - & $\mathrm{X}$ & - \\
\hline Madrid, C. & - & $\mathrm{X}$ & - & - \\
\hline Murcia, R. & - & - & - & - \\
\hline Navarra, C.F. & $\mathrm{X}$ & $\mathrm{X}$ & - & $\mathrm{X}$ \\
\hline País Vasco & - & - & \\
\hline Rioja, La & & $\mathrm{X}$ & - & \\
\hline
\end{tabular}

archivos para mostrar la información sobre sus sistemas de archivos y otras optan por cumplimentar información general sobre la valoración en el portal y a través de enlaces a las páginas de archivos. No obstante, hay que señalar que la cantidad y exhaustividad de los contenidos de las webs de transparencia sobre valoración documental es mucho menor que los de las webs de archivos o de las comisiones calificadoras, como se ha explicado con anterioridad. La excepción es el Principado de Asturias que, solo publica la documentación de valoración en el portal de transparencia.

En la figura 2 se muestra el porcentaje de páginas que contienen documentos de valoración documental de las distintas CC.AA.

Figura 2. Páginas Web con documentos de valoración documental.

$$
\text { — PORCENTAJE SÍ = PORCENTAJE NO }
$$

EN WEB COMISIÓN CALIFICADORA

EN WEB ARCHIVOS

REPARTIDO ENTRE AMBAS WEBS

EN WEB TRANSPARENCIA

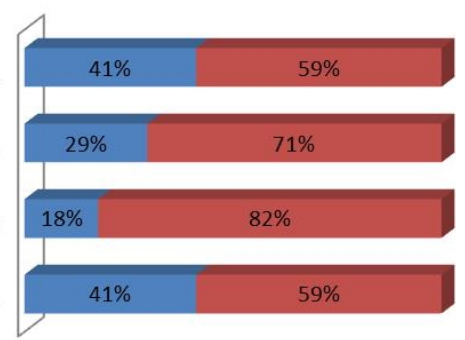

\subsection{Publicación de los documentos}

El resultado del análisis muestra que solo la Comunidad de Andalucía ofrece la información de las tablas de valoración de series documentales en formatos reutilizables siguiendo las recomendaciones sobre datos abiertos. En cuanto a la presentación de la información estructurada o tabulada, diez de las páginas de archivos, es decir, el 59\% del total, presentan los calendarios de conservación en forma de tabla o con campos estructurados, el resto presentan las series documentales valoradas en formato de lista. En la Tabla III se muestran las CC.AA. que cumplen con este parámetro y los siguientes que se analizan en este apartado.

También se presenta información a través de enlaces a otros documentos que permiten acceder a más información sin visualizarla directamente. En trece de las páginas de archivos, es decir el $76 \%$ del total, se puede acceder a través de los enlaces que hay en los calendarios de conservación, tanto a estudios de identificación y valoración correspondientes, como a los boletines oficiales donde se publican los acuerdos de aprobación.

Sobre la utilización de sistemas de búsqueda de información, cinco de las páginas de archivos, es decir, el $29 \%$ del total, los tienen para la búsqueda de series documentales valoradas, y algunas, como Castilla y León y Castilla La Mancha, también utili- 
Tabla III. Presentación de la información de valoración en las páginas Web de las Comunidades Autónomas

\begin{tabular}{|l|c|c|c|c|c|c|}
\hline & $\begin{array}{c}\text { FORMATOS } \\
\text { REUTILIZABLES }\end{array}$ & $\begin{array}{c}\text { INFORMACIÓN } \\
\text { ESTRUCTURADA }\end{array}$ & $\begin{array}{c}\text { INFORMACIÓN } \\
\text { POR ENLACES }\end{array}$ & $\begin{array}{c}\text { SISTEMAS } \\
\text { BÚSQUEDA }\end{array}$ & $\begin{array}{c}\text { SISTEMAS DE } \\
\text { ORDENACIÓN }\end{array}$ & $\begin{array}{c}\text { ACTUALIZACIÓN } \\
\text { WEB }\end{array}$ \\
\hline Andalucía & $\mathrm{X}$ & $\mathrm{X}$ & $\mathrm{X}$ & $\mathrm{X}$ & - & $\mathrm{X}$ \\
\hline Aragón & - & $\mathrm{X}$ & $\mathrm{X}$ & - & - & $\mathrm{X}$ \\
\hline Asturias, P. & - & - & - & - & - & - \\
\hline Balears, I. & - & $\mathrm{X}$ & $\mathrm{X}$ & $\mathrm{X}$ & - & $\mathrm{X}$ \\
\hline Canarias & - & - & $\mathrm{X}$ & - & - & - \\
\hline Cantabria & - & - & - & - & - & - \\
\hline Castilla y León & - & $\mathrm{X}$ & - & $\mathrm{X}$ & - & - \\
\hline Castilla La Mancha & - & $\mathrm{X}$ & $\mathrm{X}$ & - & - & - \\
\hline Cataluña & - & - & $\mathrm{X}$ & $\mathrm{X}$ & $\mathrm{X}$ & - \\
\hline C. Valenciana & - & $\mathrm{X}$ & $\mathrm{X}$ & $\mathrm{X}$ & - & - \\
\hline Extremadura & - & $\mathrm{X}$ & $\mathrm{X}$ & - & - & - \\
\hline Galicia & - & - & $\mathrm{X}$ & - & - & - \\
\hline Madrid, C. & - & $\mathrm{X}$ & $\mathrm{X}$ & - & - & - \\
\hline Murcia, R. & - & - & - & - & - & - \\
\hline Navarra, C.F. & - & - & $\mathrm{X}$ & - & - & - \\
\hline País Vasco & - & $\mathrm{X}$ & $\mathrm{X}$ & - & - & - \\
\hline Rioja, La & - & $\mathrm{X}$ & $\mathrm{X}$ & - & - & - \\
\hline
\end{tabular}

Figura 3. Presentación de la información en las páginas Web de archivos,

\section{- PORCENTAJE SÍ \#PORCENTAJE NO}

FORMATOS REUTILIZABLES

INF. TABULADA O ESTRUCTURADA

INF. A TRAVÉS DE ENLACES

SISTEMAS DE BÚSQUEDA

SISTEMAS DE ORDENACIÓN

ACTUALIZACIÓN PÁGINA

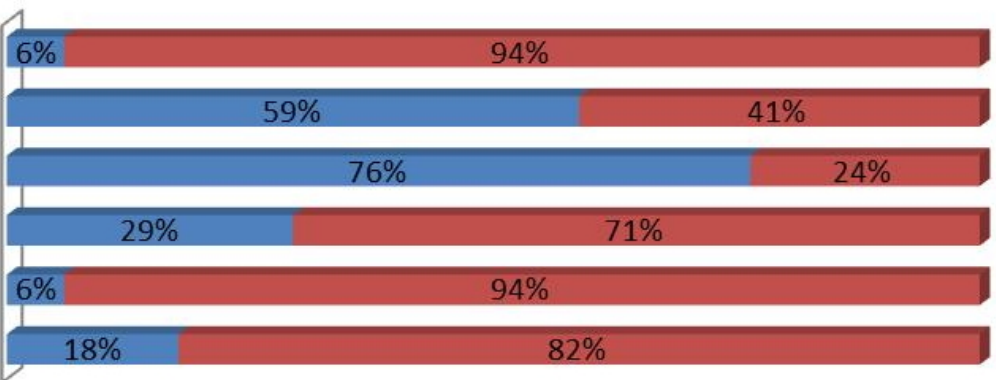

zan filtros de búsqueda por organismo productor o dictamen. Los sistemas de ordenación para ayudar a las búsquedas solo se utilizan en Cataluña.

Por último, se quiere comprobar si la información publicada en la web está actualizada y para ello se analiza si aparece junto a los calendarios de conservación la fecha de actualización. Sólo en tres de las páginas, es decir, el $18 \%$ del total, aparece de forma visible la fecha de publicación o actualización.

En la figura 3 se muestra el porcentaje de páginas de archivos que cumplen con los parámetros estudiados sobre la presentación de la información.

\section{CONCLUSIONES}

Después de analizar todas las páginas web objeto de estudio para comprobar cómo se está llevando a cabo el proceso de la valoración en la administración de las Comunidades Autónomas españolas y cómo se está difundiendo todo su trabajo, se pueden enumerar las siguientes conclusiones:

- El grado de desarrollo de la valoración documental es muy dispar entre las Comunidades Autónomas. Desde las que tienen un sistema consolidado y normalizado en sus sistemas de 
archivos, hasta las que no tienen el sistema de valoración en funcionamiento.

- No se aprecia un objetivo claro de transparencia del proceso en su conjunto, pues la mayor parte de los documentos publicados en las webs de archivos son los calendarios de conservación, que ya se publican por obligación en los boletines oficiales correspondientes.

- Los documentos que contienen las normas y los criterios que se tienen en cuenta para tomar las decisiones de valoración se publican en muy pocas Comunidades Autónomas, por lo que puede transmitirse cierta inseguridad del proceso al desconocer el motivo por el cual se toman unas decisiones y no otras en la valoración documental.

- La mayor parte de la documentación publicada de las comisiones calificadoras es la relativa a su composición y funcionamiento, pero la mayoría de las Comunidades no publican las actas de sus reuniones.

- Los calendarios de conservación de las comunidades autónomas, aun siendo los principales documentos de la valoración documental, se muestran en una gran disparidad de formatos y utilizando muy variada terminología.

- Prácticamente no se publican, excepto contadas excepciones, los documentos que recogen la información sobre la aplicación de los dictámenes tomados, es decir, no se hace público qué documentos se eliminan y cuáles se conservan. La transparencia debería ser total para la identificación de los documentos destruidos y se deberían publicar no solo datos cuantitativos, como sucede en algunos casos.

- La información sobre la valoración documental de las CC.AA. puede estar disponible en diferentes páginas de la institución, lo que puede ser un obstáculo para su localización y actualización.

- La forma en que se publican los documentos de la valoración en las páginas web no contribuye a su accesibilidad o reutilización, pues la gran mayoría de la información tiene formato Pdf o Word. Los sistemas de búsqueda también están poco implantados.

- En muchas páginas se aprecia claramente que el objetivo de la publicación de la información sobre valoración documental es explicar el procedimiento y proporcionar los formularios necesarios para su tramitación. Es decir, es información que se publica solo para uso interno o como recurso estrictamente profesional.
Muchas de estas conclusiones ya se apreciaban en el trabajo del Grupo de Archiveros Municipales de la Comunidad de Madrid (2013) como la falta de normalización terminológica o la escasez de estudios de identificación y valoración, por lo que se ha avanzado poco. También, tiene coincidencias con una de las conclusiones del trabajo sobre transparencia y acceso a los proyectos de investigación en las universidades públicas españolas (Pacios y otros, 2016), en concreto en la de que casi nunca se publican los resultados de los proyectos de investigación, al igual que los resultados de la valoración documental.

\subsection{Recomendaciones}

En vista de esta situación se proponen las siguientes recomendaciones:

- Los profesionales de los archivos deberían tomar conciencia de la importancia de la transparencia del trabajo, máxime en procesos archivísticos como la valoración documental. Se debería publicar toda la documentación de la valoración documental para aportar confianza y seguridad y así contribuir a la transparencia de las organizaciones y al buen gobierno.

- Se deberían establecer criterios generales comunes para la valoración, y de este modo evitar diferentes criterios según la Comunidad Autónoma. Se podría hacer un análisis comparativo de los criterios publicados en todas las CC.AA. para presentar una propuesta única.

- La normalización terminológica es fundamental, y más aún tras la obligación de la implantación de la administración electrónica, en la que intervienen actores con diferentes perfiles profesionales, como los tecnológicos o jurídicos, a los que se les debe transmitir una consistencia en los términos de los procesos archivísticos.

- En la medida de lo posible los diferentes sistemas podrían ir adaptando su terminología a la empleada en el Esquema de Metadatos para la gestión del documento electrónico, norma de obligado cumplimiento y de rango nacional (MINHAP, 2016a).

- Se deberían analizar todos los calendarios de conservación de las Comunidades Autónomas para comprobar, en primer lugar, la terminología empleada y, en segundo lugar, los dictámenes aprobados para series similares para comprobar si hay uniformidad en las propuestas de selección. 
- La comunidad archivística debería reflexionar sobre cómo quiere comunicarse con la ciudadanía a través de la web. Se deberían establecer criterios para establecer qué información sobre el trabajo que se realiza en los diferentes sistemas de archivos se deben transmitir y realizar estudios de usuarios para saber qué les interesa.

- Los portales de transparencia pueden servir para reenviar a las páginas de los sistemas de archivos, que es donde la información está en su contexto. Pero también deberían aportar algo más que un aumento de la visibilidad, mejorando la calidad de la información.

- Debería valorarse la posibilidad de crear redes de colaboración para afrontar los trabajos de valoración de forma conjunta, repartiendo esfuerzos y evitando diversidades de criterios.

- Por último, debería tenerse en cuenta que la gestión documental es una herramienta funda-

\section{REFERENCIAS}

Cascudo-Rasilla, A. (2018). Procedimiento administrativo común, valoración de series singular. Boletín $A N A B A D$, 3-4, 95-112.

Casellas i Serra, L.E. (2001). Evaluación y selección de la documentación económica del municipio: la experiencia desde Cataluña. En: Asociación para la Defensa del Patrimonio Bibliográfico y Documental de Cantabria (ed.), III Jornadas de Archivos Municipales de Cantabria, pp.123-163. Santander.

Casellas i Serra, L.E. (2014). Funciones y valores en la evaluación de documentos municipales: ¿del discurso al método o del método a la metodología? Comma, 1-2, 153-166. Disponible en: http://www.girona.cat/ sgdap/docs/CASELLAS_2014_Comma.pdf [Fecha de consulta: 10/02/2019].

Casellas i Serra, L.E. (2017a). Destrucción de documentos y transparencia: ¿Criterios más allá de la retórica? El Consultor de los Ayuntamientos. La gestión documental en la nueva administración digital, 7, 916-935. Disponible en: http://www.girona.cat/sgdap/docs/CASELLAS_2017_Destruccion-Transparencia.pdf [Fecha de consulta: 10/02/2019].

Casellas i Serra, L.E. (2017b). Evaluación archivística, más allá de evaluar para conservar y eliminar, Revista REDAR, 4(2), 49-77. Disponible en: http://www.girona.cat/sgdap/docs/CASELLAS_2018_CAM-Municipales.pdf [Fecha de consulta: 10/02/2019].

Cermeno-Martorell, L. (2003). El sistema de evaluación de documentos en Cataluña. Tabula: revista de archivos de Castilla y León, 6, 153-184.

Cermeno-Martorell, L.; Rivas-Palá, E. (2010). Valorar y seleccionar documentos. Qué es y cómo se hace. Gijón: Trea.

Cermeno-Martorell, L.; Rivas-Palá, E. (2011). Valoración y selección de documentos. En: Cruz Mundet, J.R. (Coord.), Administración de Documentos y Archivos. mental para la transparencia de las organizaciones, facilita a los ciudadanos el derecho de acceso a la información pública, proporciona confianza en las administraciones públicas y contribuye a desarrollar el buen gobierno.

\section{AGRADECIMIENTOS}

Este artículo ha sido posible gracias a las indicaciones y correcciones de María del Carmen Rodríguez López, profesora titular de la Universidad de León, Departamento de Patrimonio Artístico y Documental.

\section{AKNOWLEDGEMENTS}

This article has been possible thanks to the indications and corrections of María del Carmen Rodríguez López, full professor at the University of León, Department of Artistic and Documentary Heritage.

Textos Fundamentales, [en línea], pp. 215-271. Madrid: Coordinadora de Asociaciones de Archiveros. Disponible en: http://hdl.handle.net/10016/19730

Codina-Bonilla, L. (2000). Evaluación de recursos digitales en línea: conceptos, indicadores y métodos. Revista Española de Documentación Científica, 23(1), 9-44. https://doi.org/10.3989/redc.2000.v23.i1.315

Codina-Bonilla, L. (2018). Sistemas de búsqueda y obtención de información, componentes y evolución. Anuario ThinkEPI, 12, 77-82. https://doi.org/10.3145/ thinkepi.2018.06

Cortés-Alonso, V. (1983). Transferencias y expurgos. En: Diputación Provincial de Sevilla (ed.), Archivística, estudios básicos, pp. 145-164. Sevilla.

Couture, C. (2003). La función valoración en la archivística contemporánea: una sinergia entre varias consideraciones complementarias. Tabula: Revista de Archivos de Castilla y León, 6, 23-49.

Cruz-Mundet, J.R. (1994). Manual de Archivística. Madrid: Fundación Germán Sánchez-Ruipérez.

Cruz-Mundet, J.R. (2006). La gestión de documentos en las organizaciones. Madrid: Pirámide.

Cruz-Mundet, J.R. (2012). Archivística. Gestión de documentos y administración de archivos. Madrid: Alianza Editorial.

España. (1985). Ley 16/1985, de 25 de junio, del Patrimonio Histórico Español. BOE n. 155, 29 junio 1985. Disponible en: https://www.boe.es/eli/ es/l/1985/06/25/16/con

España. (2007a). Ley 11/2007, de 22 de junio, de acceso electrónico de los ciudadanos a los Servicios Públicos, BOE n. 150, 23 junio 2007. Disponible en: https:// www.boe.es/eli/es/l/2007/06/22/11

España. (2007b). Ley 37/2007, de 16 de noviembre, sobre reutilización de la información del sector público y su normativa de desarrollo, BOE n. 276, 17 no- 
viembre 2007. Disponible en: https://www.boe.es/eli/ es/l/2007/11/16/37/con

España. (2011). Real Decreto 1708/2011, de 18 de noviembre, por el que se establece el Sistema Español de Archivos y se regula el Sistema de Archivos de la Administración General del Estado y de sus Organismos Públicos y su régimen de acceso, BOE n. 284, 25 noviembre 2011. Disponible en: https://www.boe.es/ eli/es/rd/2011/11/18/1708/con

España. (2013). Ley 19/2013, de 9 de diciembre, de transparencia, acceso a la información pública y buen gobierno, BOE n. 295, 10 diciembre 2013. Disponible en: https://www.boe.es/eli/es/l/2013/12/09/19

España. (2015). Ley 39/2015, de 1 de octubre, del Procedimiento Administrativo Común de las Administraciones Públicas, BOE n. 236, 2 octubre 2015. Disponible en: https://www.boe.es/eli/es/l/2015/10/01/39

Fernández-Cuervo, C. (1996). Valoración, transferencias y expurgo en los archivos judiciales. En: Diputación General de Aragón, Departamento de Educación y Cultura (ed.), Actas de las VI Jornadas de Archivos Aragoneses: situación y perspectivas de los archivos de la administración de justicia, pp. 245-256. España.

Fernández-Cuesta, F. (2018). ISO/TR 21946:2018. Valoración para la gestión de documentos. Sensaciones encontradas. Archivamos/Boletín ACAL: Revista Trimestral de la Asociación de Archiveros de Castilla y León, 110, 40-41.

Garmendia-Ruíz, B. (2000). La valoración documental en el sistema archivístico de Castilla y León. Subsistema de la documentación autonómica. Boletín ACAL, $10(35), 29-31$.

Gómez-Loeches, L. (1997). El expurgo en los archivos judiciales. En: Junta de Comunidades de Castilla-La Mancha (ed.), La administración de justicia en la historia de España: actas de las III Jornadas de Castilla-La Mancha sobre Investigación en Archivos, pp. 829-850. España: Guadalajara.

González-Cachafeiro, J. (Coord.). (2013). 6a Jornadas Archivando: la valoración documental. León: Fundación Sierra Pamblei. Disponible en: https://dialnet.unirioja. es/servlet/libro?codigo $=571584$ [Fecha de consulta: 10/02/2019]

Grupo de Archiveros Municipales de Madrid. (1990). El expurgo en los archivos municipales, propuesta de un manual, En: Comunidad de Madrid; Ayuntamiento de Leganés (eds.), VII Jornadas de Archivos Municipales, Leganés. Madrid. Disponible en: http://www.madrid. org/archivos/images/ACTIVIDADES/PUBLICACIONES/VIIjarchivosmunicipales.pdf [Fecha de consulta: 12/03/2019].

Grupo de Archiveros Municipales de Madrid. (2013). El gran reto de la archivística contemporánea: La Valoración. En: Comunidad de Madrid; Ayuntamiento de Torremolinos (eds.), Actas de las XIX Jornadas de Archivos Municipales. Gestión documental: soluciones para la e-Administración, pp. 91-205. Madrid. Disponible en:http://www.madrid.org/archivos/images/ACTIVIDADES/PUBLICACIONES/XIXjarchivosmunicipales.pdf [Fecha de consulta: 05/03/2019].

Heredia-Herrera, A. (1986). Archivística general, teoría y práctica. Sevilla: Servicio de publicaciones de la Diputación de Sevilla.
Heredia-Herrera, A. (2013). Manual de archivística básica: Gestión y Sistemas. México: BUAP.

Hernández-Olivera, L. (2005). Todo lo que usted quería saber sobre teoría de la valoración. Archivamos: Boletín $A C A L, 57-58,39-40$.

Hernández-Olivera, L. (2008). El valor del documento electrónico. Archivamos: Boletín ACAL, 68, 19-20.

Hernández-Olivera, L. (2013). Sobre la constitución de la memoria social. Hacia una valoración de documentos postmoderna. En: González-Cachafeiro, J. (Coord.), 6as Jornadas Archivando: la valoración documental: actas de las jornadas. León, Fundación Sierra Pambley, pp. 5-20. Disponible en: https://dialnet.unirioja.es/servlet/ libro?codigo=571584 [Fecha de consulta: 24/02/2019].

Hernández-Olivera, L.; Moro-Cabero, M. (1997). El proceso de valoración y selección documental. Metodología para su aprendizaje. En: Martínez Comeche, J.A. (coord.), Métodos didácticos en Biblioteconomía y Documentación. VI Jornadas Académicas organizadas por la Escuela Universitaria de Biblioteconomía y Documentación de la Universidad Complutense de Madrid, pp. 279-295. Madrid: Universidad Complutense de Madrid.

Hernández-Olivera, L.; Moro-Cabero, M. (2002a). La destrucción de documentos de la administración estatal: comentarios sobre su regulación. Archivamos: Boletín $A C A L, 45-46,19-28$

Hernández-Olivera, L.; Moro-Cabero, M. (2002b). Procedimientos de valoración documental. Salamanca: Acal.

Hernández-Olivera, L.; Moro-Cabero, M. (2003). Seleccionar documentos con el calendario de conservación: una propuesta para Castilla y León. Tabula: Revista de Archivos de Castilla y León, 6, 121-152.

Hernández-Olivera, L.; Moro-Cabero, M. (2009). Determinando la memoria: la valoración de documentos. En: Sendín García, M.N.; Gómez Díaz, R. (coords.), Régimen jurídico de los documentos: aspectos administrativos, civiles, penales y procesales, pp. 327-356. España: Comares.

ISO/TR 21946: 2018. Information y Documentation: Appraisal for managing records. Ginebra: ISO.

La Torre-Merino, J.L. (2000). Metodología para la identificación y valoración de fondos documentales. Madrid: Ministerio de Educación, Cultura y Deporte.

Lodolini, E. (1984). Archivística. Principi e problemi. Milán: Franco Angeli.

Ministerio de Cultura. (1991). Actas de las Primeras Jornadas sobre Metodología para la Identificación y Valoración de Fondos Documentales de las Administraciones Públicas. Madrid: Dirección General de Bellas Artes y Archivos.

Ministerio de Cultura y Deporte. (1995). Diccionario de terminología archivística. Normas técnicas de la Subdirección General de Archivos Estatales, [en línea]. Madrid. Disponible en: http://www.culturaydeporte. gob.es/cultura/areas/archivos/mc/dta/portada.html [Fecha de consulta: 19/02/2019].

Ministerio de Hacienda y Administraciones Públicas, (MINHAP). (2016a). Esquema de metadatos para la gestión del documento electrónico (e-EMGDE), Documentación complementaria a la Norma Técnica de Interoperabili- 
dad de Política de gestión de documentos electrónicos (versión 2.0). Madrid. Disponible en: https://administracionelectronica.gob.es/pae_Home/pae_Estrategias/ pae_Interoperabilidad_Inicio/pae_Normas_tecnicas_ de_interoperabilidad.html\#POLITICAGESTION [Fechā de consulta: 10/04/2019].

MINHAP (2016b). Ministerio de Hacienda y Administraciones Públicas, Política de gestión de documentos electrónicos. Guía de aplicación de la Norma Técnica de Interoperabilidad. Madrid. Disponible en: https://administracionelectronica.gob.es/pae_Home/pae_Estrategias/pae_Interoperabilidad_Inicio/pae_Normas_tecnicas_de_interoperabilidad.html\#POLITICAGESTION [Fecha de consulta: 10/05/2019].

Pacios, A.R., Vianello-Osti, M.; Rodríguez-Bravo, B. (2016). Transparencia y acceso a la información sobre los proyectos de investigación en las universidades públicas españolas. El Profesional de la Información, 25(5), 721-729. https://doi.org/10.3145/epi.2016. sep.02.

Páez-García, M.A. (2010). La valoración y selección documental en Andalucía: pasado, presente y futuro. $R e$ vista TRIA, 16, 73-104. Disponible en: https://dialnet. unirioja.es/servlet/articulo?codigo $=3864015$ [Fecha de consulta: 10/05/2019].

Pérez-Santana, J.F. (2018). La estadística de archivos en el marco de la Transparencia. Radiografía de 2017. En: Andrés Díaz, R.; Sierra Rodríguez, J. (coords.), La función de archivo y la transparencia en España. Madrid: Universidad Complutense de Madrid. pp. 99-

124. Disponible en: https://eprints.ucm.es/49605/1/ LIBRO $\% 202018 \% 20$ LA $\% 2$ F FUNCI\%C3\%93N\%20 DE\%20ARCHIVO\%20Y\%20LA\%20TRANSPARENCIA. pdf [Fecha de consulta: 10/02/2019].
Rivas-Palá, E. (2003). Identificación, valoración y selección de series documentales de obras y urbanismo. En: Asociación para la Defensa del Patrimonio Bibliográfico y Documental de Cantabria (ed.), IV Jornadas de Archivos Municipales de Cantabria, pp. 31-72. Santander.

Rivas-Palá, E. (2013). La valoración documental en el entorno de la administración electrónica. En: González-Cachafeiro, J. (Coord.), Gas Jornadas Archivando: la valoración documental: actas de las jornadas. León, Fundación Sierra Pambley, pp. 21-41. Disponible en: https://dialnet.unirioja.es/servlet/libro?codigo=571584 [Fecha de consulta: 12/03/2019].

Ruiz-Cagigal, A. (2017). La solución de las comisiones calificadoras de documentos administrativos. El Consultor de los Ayuntamientos, 14, 1.816-1.831.

Schellenberg, T.R. (1958). Archivos Modernos. La Habana: imprenta del Archivo Nacional.

Sorli-Rojo, A.; Mochón-Bezares, G. (2002). Tesauro de Biblioteconomía y Documentación, [en línea]. España: CSIC-CINDOC. Disponible en: http://hdl.handle. net/10261/30255 [Fecha de consulta: 12/03/2019].

Transparency International España. (2016). Índice de transparencia de las comunidades autónomas 2016. España. Disponible en: https://transparencia.org.es/ wp-content/uploads/2016/12/cuadro indicadores incau-2016.pdf [Fecha de consulta: 27/02/2019].

UNE-ISO 15489-1 (2006). Información y documentación. Gestión de documentos. Madrid: AENOR

UNE-ISO 15489-1 (2016). Información y documentación. Gestión de documentos. Madrid: AENOR

Vázquez-Murillo, M. (1995). Manual de selección documental. Carmona: S\&C Ediciones. 